\title{
Frühes Stadium beim nichtkleinzelligen Lungenkarzinom
}

\author{
Wilfried Eberhardt ${ }^{a *}$ Frank Griesinger ${ }^{\mathrm{b} *}$ Martin Filipits ${ }^{\mathrm{c} *}$ Hauke Winter ${ }^{\mathrm{d} *}$ \\ ${ }^{a}$ Innere Klinik - Tumorforschung, Westdeutsches Tumorzentrum, Universitätsklinikum Essen, Universität Duisburg-Essen, Essen, \\ ${ }^{\mathrm{b}}$ Klinik für Hämatologie und Onkologie, Pius-Hospital Oldenburg, Deutschland \\ c Institut für Krebsforschung, Medizinische Universität Wien, Österreich \\ ${ }^{d}$ Chirurgische Klinik und Poliklinik, Klinikum der Universität München, Campus Großhadern, München, Deutschland
}

\begin{abstract}
Ausmaß der Resektion im Stadium la - wie viel ist nötig?
Patienten im Tumorstadium Ia haben mit einer 5-Jahres-Überlebensrate von mehr als 70\% eine sehr gute Prognose. Die besten Ergebnisse hinsichtlich des rezidivfreien und Gesamtüberlebens sind bei einer Lobektomie mit mediastinaler und hilärer Lymphknotendissektion zu erwarten. Für die «klassische» Resektion sind allerdings nur Patienten mit ausreichender kardiopulmonaler Reserve geeignet; bei Patienten mit entsprechenden Einschränkungen werden in Kombination mit Lymphadenektomie meist limitierte Resektionen, wie Segment-Resektion, Wide-Exzision und Wedge-Resektion, durchgeführt. Zur Wirksamkeit dieser Verfahren liegen fast nur Daten aus retrospektiven Studien vor. Diese zeigen zwar, dass Patienten auch von einer limitierten Resektion profitieren können, ermöglichen aber keine Beurteilung im Vergleich zur Lobektomie.
\end{abstract}

\section{Lobektomie versus limitierte Resektionen}

In der bisher einzigen abgeschlossenen, randomisierten Vergleichsstudie untersuchte die US-amerikanische Lung Cancer Study Group zwischen 1982 und 1988 bei Patienten mit Tumoren $\leq 3 \mathrm{~cm}(\mathrm{~N} 0)$ die Lobektomie $(\mathrm{n}=125)$ und limitierte Resektionen (Segmentresektion $\mathrm{n}=82$; Wedge-Resektion $\mathrm{n}=40$ ) hinsichtlich des rezidivfreien und Gesamtüberlebens. Nach einem Follow-up von mindestens 4,5 Jahren war die Rezidivrate bei Patienten mit limitierter Resektion signifikant höher ( $\mathrm{p}=0,016)$; das Gesamtüberleben war dagegen in den ersten 4 Jahren vergleichbar und bei längerer Beobachtungsdauer nicht signifikant schlechter als bei Lobektomie $(\mathrm{p}=0,088)$ [1]

Bei der Bewertung dieses Ergebnisses ist zu berücksichtigen, dass es sich um Daten aus den 1980er-Jahren handelt und dass das Krankheits-Staging inzwischen mittels besserer CT-

*Alle Autoren haben zu gleichen Teilen zu diesem Artikel beigetragen.
Untersuchungen sowie PET-CT und endobronchialem Ultraschall (EBUS) deutlich verbessert worden ist.

Eine Metaanalyse, in der bei Patienten mit Tumoren $\leq$ $3 \mathrm{~cm}$ bei Lobektomie und limitierter Resektion das Überleben nach 1, 3 und 5 Jahren untersucht wurde, zeigte für die Lobektomie erst nach einem längeren Follow-up einen größeren, aber nicht signifikanten Vorteil im Überleben [2].

\section{Einfluss von Tumorgröße, Sicherheitsabstand, Lymphknoten und Histologie}

Der Einfluss der Tumorgröße auf die Prognose bei limitierter Resektion wurde retrospektiv anhand der Aufzeichnungen von Patienten mit kompletter Resektion eines nichtkleinzelligen Lungenkarzinoms (NSCLC) im Stadium pT1 untersucht. Während bei kleinen Tumoren $(\leq 20 \mathrm{~mm})$ nicht nur bei Segment-Resektion, sondern auch bei Wedge-Resektion eine hohe krankheitsspezifische 5-Jahres-Überlebensrate beobachtet wurde (97,7\% vs. 85,7\%), war die Segment-Resektion der Wedge-Resektion bei größeren Tumoren (21-30 mm bzw. >30 mm) deutlich überlegen ( $84,6 \%$ vs. $39,4 \%$ bzw. $62,9 \%$ vs. $0 \%$ ) [3].

Die Bedeutung des Sicherheitsabstands für die Prognose bei limitierter Resektion verdeutlichte eine japanische Studie. Von den Patienten, deren Tumor mit einem Sicherheitsabstand von $>20$ mm oder einem den größten Tumordurchmesser übertreffenden Abstand reseziert worden war, entwickelte keiner ein Rezidiv, bei einem kleineren Sicherheitsabstand waren dagegen $13,4 \%$ bzw. $14 \%$ betroffen [4].

Den Einfluss der Lymphknotendissektion auf die Prognose zeigte eine retrospektive Studie aus Boston, in der bei Patienten mit Tumoren $\leq 2 \mathrm{~cm}$ die Lobektomie $(\mathrm{n}=84)$ mit limitierten Resektionen $(\mathrm{n}=154)$ verglichen wurde. Die Lobektomie war in dieser Studie mit einem signifikant besseren Gesamtüberleben ( $p=0,0027)$ assoziiert als bei den Patienten, die eine limitierte Resektion ohne zusätzliche Lymphadenektomie erhielten; bei Patienten mit Lymphknoten-Sampling wurde aber auch bei limitierter Resektion ein vergleichbar gutes Gesamtüberleben wie bei Patienten mit Lobektomie erreicht [5].

\begin{tabular}{ll}
\hline KARGER & @ 2012 S. Karger GmbH, Freiburg \\
0378-584X/12/3515-0006 \$38.00/0 \\
$\begin{array}{l}\text { Fax+497614520714 } \\
\text { Information@Karger.de } \\
\text { www.karger.com }\end{array}$ & $\begin{array}{l}\text { Accessible online at: } \\
\text { www.karger.com/onk }\end{array}$
\end{tabular}


Auch beim prognostisch günstigen Adenokarzinom in situ (AIS), minimal invasiven Adenokarzinom sowie bei kleinen Adenokarzinomen mit überwiegend lepidischem Wachstumsmuster wurden bei limitierten Resektionen sehr gute Therapieergebnisse mit Raten für das krankheitsfreie Überleben (disease free survival; DFS) von 100\% berichtet [6].

\section{Stadium Ib-IIla: Adjuvant versus neoadjuvant}

\section{Adjuvante Therapie}

Nach vollständiger Resektion eines NSCLC im Stadium II und III gilt die adjuvante Chemotherapie mit einem Cisplatinhaltigen Schema heute als Therapiestandard und wird als individuelles Therapiekonzept auch im Stadium IB erwogen. Zurückzuführen ist dies auf die Ergebnisse der randomisierten Studien IALT, JBR.10 und ANITA [7-9], in denen die adjuvante Therapie mit einer Cisplatin-haltigen Dublette das mediane Gesamtüberleben und DFS statistisch signifikant verlängerte.

\section{Metaanalyse LACE}

Ergänzt wurden diese Daten durch die Ergebnisse der Metaanalyse LACE (Lung Adjuvant Cisplatin Evaluation), in der die Daten von insgesamt 4584 Patienten ausgewertet wurden, die in den Studien IALT, JBR.10 und ANITA sowie ALPI und BLT eine adjuvante Chemotherapie erhielten oder der Beobachtungsgruppe zugeteilt waren [10].

Nach einem medianen Follow-up von 5,2 Jahren war bei den adjuvant behandelten Patienten eine signifikante Abnahme des Mortalitätsrisikos von $11 \%$ zu sehen (Hazard Ratio (HR) 0,89; 95\%-Konfidenzintervall (KI) 0,82-0,96; p = 0,005), entsprechend einem absoluten 5-Jahres-Überlebensvorteil von 5,4\% (Tab. 1). Der absolute Vorteil im DFS betrug nach 5 Jahren 5,8\%, die HR 0,84 (95\%-KI 0,78-0,91; p < 0,001).

Wie eine Analyse in Abhängigkeit vom Tumorstadium zeigte, hatten Patienten im Stadium II und III einen statistisch signifikanten Benefit von der adjuvanten Therapie, bei Patienten mit IB-Tumoren war nur ein positiver Trend zu sehen; dies ist am ehesten durch die nicht ausreichende Zahl an Patienten in dieser Gruppe zu erklären. Patienten mit Tumoren im Stadium IA, die allerdings nur einen kleinen Anteil des Gesamtkollektivs darstellten, hatten keinen Nutzen von der adjuvanten Chemotherapie.

Einen vergleichbaren Überlebensvorteil der adjuvanten Chemotherapie zeigte eine weitere Metaanalyse, die etwa 8450 Patienten aus 34 Studien einschloss. In diesem Kollektiv verringerte die postoperative Chemotherapie das Mortalitätsrisiko um 14\% (HR 0,86; 95\%-KI 0,81-0,92; p < 0,0001), wobei das 5-Jahres-Überleben von 60\% (Kontrolle) auf 64\% stieg [11].

Neben der signifikanten Verbesserung der Prognose generiert die primäre Operation eine Resektatpathologie, die mit hoher Präzision die Prognose vorhersagt und Voraussetzung
Tab. 1. Metaanalyse LACE: Gesamtüberleben (modifiziert nach [10])

\begin{tabular}{lccc}
\hline Studie & Ereignisse & Patienten & HR $(95 \%-K I)$ \\
\hline ALPI & 569 & 1088 & $0,95(0,81-1,12)$ \\
ANITA & 458 & 840 & $0,82(0,68-0,98)$ \\
BLT & 186 & 307 & $0,95(0,71-1,27)$ \\
IALT & 980 & 1867 & $0,91(0,81-1,04)$ \\
JBR.10 & 197 & 482 & $0,71(0,54-0,94)$ \\
Gesamt & 2390 & 4584 & $0,89(0,82-0,96)$ \\
\hline
\end{tabular}

für die Etablierung von für die adjuvante Therapie prädiktiven «Biomarkern» ist. Ein potenzieller Nachteil ist dagegen die im Vergleich zur neoadjuvanten Therapie durchweg etwas ungünstigere Therapieadhärenz. Besonders bei Patienten mit Pneumonektomie muss bei einer postoperativen Chemotherapie prinzipiell mit einer erhöhten Toxizität gerechnet werden.

\section{Neoadjuvante Therapie}

Patienten mit frühem NSCLC im Stadium IB bis IIIA-3U (IIIA mit präoperativ bekanntem Unilevel-N2-Lymphknoten $>1 \mathrm{~cm}$ ) kommen prinzipiell auch für eine neoadjuvante Therapie infrage; im Vergleich zur adjuvanten Therapie bestehen dabei folgende potenzielle Vor- und Nachteile:

- Vorteile

- bessere Therapieadhärenz mit der Chemotherapie

- höhere Dosierung der Chemotherapie

- mögliches Down-Sizing und Down-Staging

- frühzeitig effektive Therapie von Mikrometastasen

- optimales Patientenkollektiv für die Evaluierung neuer Substanzen

- Nachteile

- kein pathologisches Staging möglich

- Angst des Patienten vor primärer Progression.

Daten zur Wirksamkeit, Verträglichkeit und Compliance einer neoadjuvanten Chemotherapie lieferten die Phase-III-Studien S9900, ChEST und NATCH, die bei relativ kleinen Patientenzahlen allerdings alle vorzeitig abgebrochen wurden.

S9900: Paclitaxel/Carboplatin $\rightarrow$ Operation versus Operation In der Phase-III-Studie S9900 erhielten 354 Patienten im Stadium IB-IIIA (ohne N2-Befall), darunter etwa 30\% im Stadium IIB/IIIA, als Induktionstherapie 3 Zyklen Paclitaxel/ Carboplatin. Im Vergleich zur alleinigen Operation verringerte die präoperative Chemotherapie das Progressionsrisiko und das Sterberisiko um 20\% bzw. 21\% (HR 0,80; p = 0,10 bzw. HR 0,79; $\mathrm{p}=0,11$; alle Werte nicht signifikant). Bei einer sehr hohen Therapieadhärenz der präoperativen Chemotherapie (97\% der Patienten erhielten mindestens eine Dosis, $79 \%$ alle 3 vorgesehenen Zyklen) betrug die R0-Resektionsrate $84 \%$ und lag damit im selben Bereich wie bei alleiniger Operation (87\%) [12]. 
ChEST: Cisplatin/Gemcitabin $\rightarrow$ Operation versus Operation Die ChEST-Studie, in die 270 Patienten im Stadium IB-IIIA aufgenommen wurden, darunter in beiden Armen fast 50\% im Stadium IIB/IIIA, zeigte für die präoperative Therapie mit 3 Zyklen Cisplatin/Gemcitabin einen signifikanten Vorteil sowohl im progressionsfreien Überleben (HR 0,70; $p=0,003$ ) als auch im Gesamtüberleben (HR 0,63; $\mathrm{p}=0,02$ ). Patienten im Stadium IIB/IIIA profitierten mit einer Abnahme des Progressionsrisikos um 49\% (HR 0,51; $\mathrm{p}=0,02)$ und des Sterberisikos um 58\% (HR 0,42; p < 0,001) erwartungsgemäß stärker von der Induktionstherapie als das Gesamtkollektiv. Auch in dieser Studie wurde eine sehr gute Therapieadhärenz berichtet; die R0-Resektionsrate betrug 88\% (alleinige Operation: 84\%), die perioperative Mortalität 3\% (alleinige Operation 4\%) [13].

\section{NATCH-Studie: Operation versus Paclitaxel/Carboplatin $\rightarrow$ Operation versus Operation $\rightarrow$ Paclitaxel/Carboplatin}

In der randomisierten dreiarmigen NATCH-Studie wurde bei 624 Patienten im Stadium IA (Tumorgröße $>2 \mathrm{~cm}$ ), IB, II und T3N1 die alleinige Operation mit einem neoadjuvanten und adjuvanten Therapiekonzept verglichen. Primärer Endpunkt war das DFS. Da die Randomisierung vor der Operation erfolgte, ist diese Studie die einzige Studie weltweit, die Rückschlüsse auf die Therapieadhärenz einer Induktionstherapie versus adjuvanten Therapie zulässt in einem Kollektiv, das für eine kurative Resektion vorgesehen ist. Während bei der präoperativen Therapie 97\% der Patienten mindestens 1 Dosis erhielten, begannen bei der postoperativen Therapie nur 66\% der Patienten mit der Behandlung. Der Anteil der Patienten, die die vorgesehenen 3 Zyklen erhielten, betrug 90,4\% (Induktion) bzw. 60,9\% (adjuvante Therapie) [14].

Im Vergleich zur alleinigen Operation konnte in der NATCH-Studie weder für die neoadjuvante Therapie noch für die adjuvante Therapie ein statistisch signifikanter DFSVorteil gezeigt werden (HR 0,92 bzw. 0,96). Zurückzuführen ist dies möglicherweise auf die kleinen Patientenzahlen und den mit über 70\% relativ hohen Anteils von Patienten im Stadium I. Patienten im Stadium II-T3N1 profitierten auch hier mit einer Abnahme des Progressionsrisikos um 19\% (HR 0,81; 95\%-KI 0,64-1,02) stärker als das Gesamtkollektiv.

Unter Berücksichtigung dieser Studiendaten, die bei einer im Vergleich zur adjuvanten Therapie deutlich besseren Therapieadhärenz keine Nachteile hinsichtlich der perioperativen Mortalität sowie des DFS und Gesamtüberlebens zeigten, sind neoadjuvante Therapiekonzepte eine wirksame Alternative, die vor allem bei Patienten mit erhöhtem Toxizitätsrisiko, beispielsweise bei einer vorgesehenen Pneumonektomie, sowie bei relevanten Komorbiditäten, die die Durchführung einer adjuvanten Therapie erschweren, erwogen werden können. Wegen der für die adjuvante Therapie aber klaren Präferenz für eine Cisplatin-haltige Zweierkombination sollte diese Tatsache auch bei einer möglichen neoadjuvanten Induktionschemotherapie Berücksichtigung finden. Akzeptable Kombinationen wären jene aus Cisplatin und Paclitaxel oder Cisplatin und Gemcitabin.

\section{Prognostische und prädiktive Marker bei adjuvanter Chemotherapie}

\section{IALT-Bio: ERCC1 und p27}

Im Rahmen des IALT-Bio-Projekts wurden verschiedene Transportproteine, DNA-Reparaturproteine, Zellzyklusregulatoren, Signalübertragungsproteine sowie Apoptose- und Proliferationsmarker retrospektiv auf ihre prognostische und prädiktive Wertigkeit untersucht. Als potenzieller Prädiktor für das Ansprechen auf eine adjuvante Chemotherapie wurde das DNA-Reparaturprotein ERCC1 identifiziert. Bei Patienten mit ERCC1-negativen Tumoren verlängerte die adjuvante Cisplatin-haltige Chemotherapie das Gesamtüberleben signifikant (HR 0,65; 95\%-KI 0,50-0,86; $\mathrm{p}=0,002$ ), während Patienten mit ERCC1-positiven Tumoren keinen Vorteil im Gesamtüberleben hatten (HR 1,14; 95\%-KI 0,84-1,55; $\mathrm{p}=0,40$ ) (Test auf Interaktion: $\mathrm{p}=0,009)$ [15].

Die Abwesenheit von p27 $7^{\mathrm{Kip} 1}$, einem Inhibitor Cyclin-abhängiger Kinasen, war im IALT-Bio-Projekt ebenfalls mit einem statistisch signifikanten Vorteil der adjuvanten Chemotherapie assoziiert (HR 0,66; 95\%-KI 0,50-0,88; p =0,006) (Abb. 1A) und deutete im Vergleich zu p2 $7^{\text {Kip1 }}$-positiven Tumoren bei einem im Interaktionstest gezeigten signifikanten Unterschied ( $p=0,02)$ ebenfalls auf eine prädiktive Wertigkeit hin [16].

Noch stärker war die prädiktive Wertigkeit bei Kombination beider Marker. Patienten mit doppelt negativen Tumoren hatten den größten Nutzen von der adjuvanten Chemotherapie (HR 0,52; 95\%-KI 0,36-0,74), bei Patienten mit Nachweis beider Marker war die Wirksamkeit am geringsten (Test auf Interaktion: $\mathrm{p}=0,001)$ [17].

\section{LACE-Bio: $p 27$ und KRAS}

Die im IALT-Bio-Programm gezeigte prädiktive Wertigkeit von p27 konnte im LACE-Bio-Programm, in dem Tumorproben der Studien IALT, ANITA, JBR.10 und CALGB 9633 untersucht werden, allerdings nicht bestätigt werden. Patienten mit p27-negativen Tumoren $(\mathrm{n}=356)$ hatten von der adjuvanten Chemotherapie keinen signifikanten Überlebensvorteil im Vergleich zu den allein beobachteten Patienten (HR 0,87; 95\%-KI 0,64-1,18; $\mathrm{p}=0,26)$, auch die Interaktionsanalyse der Patienten mit p27-negativen und p27-positiven Tumoren zeigte keinen signifikanten Unterschied $(p=0,83)$ [18].

\section{JBR.10: 15-Gen-Signatur}

Auf Basis der in der JBR.10-Studie gewonnen Tumorproben $(\mathrm{n}=133)$ wurde eine 15-Gen-Signatur entwickelt, die in der Beobachtungsgruppe eine Unterscheidung in Niedrigrisikound Hochrisiko-Patienten ermöglichte und prädiktiv für das Therapieansprechen war. Von einer adjuvanten Chemotherapie profitierten demnach nur Hochrisiko-Patienten (HR 0,33; 95\%-KI 0,17-0,63; p = 0,0005); Niedrigrisiko-Patienten hatten dagegen keinen Nutzen (HR 3,67; 95\%-KI 1,22-11,06; $\mathrm{p}=$ 0,0133) (Interaktionsanalyse $\mathrm{p}<0,001$ ) [19]; eine Validierung dieses Ergebnisses an einem anderen Studienkollektiv und insbesondere eine prospektive Validierung stehen aber noch aus. 


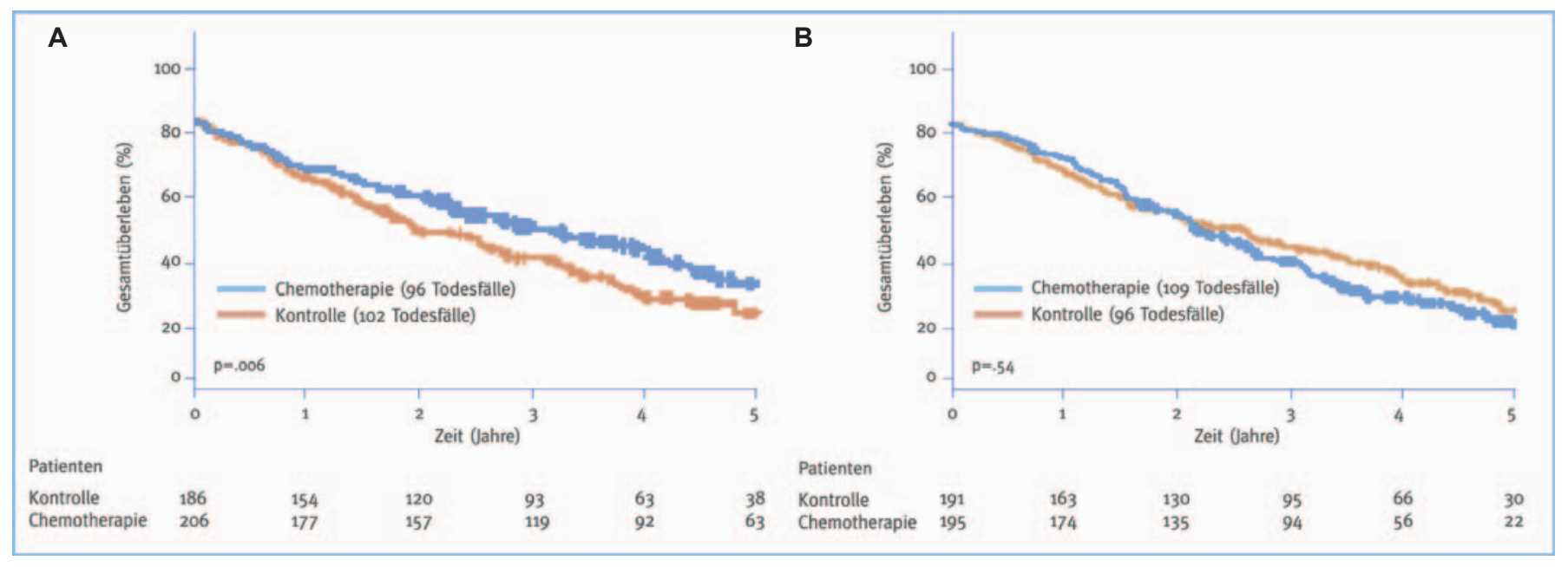

Abb. 1. IALT-Bio: Gesamtüberleben bei Patienten mit A p $27^{\text {Kip1 }}$-negativen und B p $27^{\text {Kip1 }}$-positiven Tumoren (modifiziert nach [16]).

\section{Ausblick: Biomarker-basierte Studien}

Neben weiteren retrospektiven Analysen können auch prospektive Biomarker-basierte Studien wie die SCAT- [20], die TASTE- [21] und die ITACA-Studie dazu beitragen, prädiktive Marker zur Selektion der von einer adjuvanten Chemotherapie voraussichtlich profitierenden Patienten zu identifizieren. Neben den prognostischen und prädiktiven Biomarkern müssen in Zukunft darüber hinaus noch viel mehr Daten zu Komorbiditäten und speziellen Krankheitssituationen im
Rahmen von klinischen Studien evaluiert werden. Angesichts der immer älter werdenden Patientenpopulationen und der viel breiteren Komorbiditätsprofile bei Diagnosestellung sind wir zu solchen Zusatzanalysen dringend aufgefordert.

\section{Disclosure Statement}

Die Autoren waren Referenten beim «Dresdner Interdisziplinäres Gespräch 2012», das von Roche Pharma AG unterstützt wurde.

\section{Literatur}

1 Ginsberg RJ, Rubinstein LV: Randomized trial of lobectomy versus limited resection for T1 2 N0 nonsmall cell lung cancer. Lung Cancer Study Group. Ann Thorac Surg 1995;60:615-623.

2 Nakamura H, Kawasaki N, Taguchi M, et al.: Survival following lobectomy vs limited resection for stage I lung cancer: a meta-analysis. Br J Cancer 2005;92:1033-1037.

3 Okada M, Nishio W, Sakamoto T, et al.: Effect of tumor size on prognosis in patients with non-small cell lung cancer: the role of segmentectomy as a type of lesser resection. J Thorac Cardiovasc Surg 2005;129:87-93.

4 Sawabata N, Ohta M, Matsumura A, et al.: Optimal distance of malignant negative margin in excision of non-small cell lung cancer: a multicenter prospective study. Ann Thorac Surg 2004;77:415-420.

$\checkmark 5$ Wolf AS, Richards WG, Jaklitsch MT, et al. Lobectomy versus sublobar resection for small ( $2 \mathrm{~cm}$ or less) non-small cell lung cancers. Ann Thorac Surg 2011;92:1819-1823.

-6 Travis WD, Brambilla E, Noguchi M, et al.: International Association for the Study of Lung Cancer/ American Thoracic Society/European Respiratory Society international multidisciplinary classification of lung adenocarcinoma. J Thorac Oncol 2011; 6:244-285.

7 Arriagada R, Bergman B, Dunant A, et al.: Cisplatin-based adjuvant chemotherapy in patients with completely resected non-small-cell lung cancer. N Engl J Med 2004;350:351-360.
8 Winton T, Livingston R, Johnson D, et al.: Vinorelbine plus cisplatin vs. observation in resected nonsmall-cell lung cancer. N Engl J Med 2005;352: 2589-2597.

9 Douillard JY, Rosell R, De Lena M, et al.: Adjuvant vinorelbine plus cisplatin versus observation in patients with completely resected stage IB-IIIA nonsmall-cell lung cancer (Adjuvant Navelbine International Trialist Association [ANITA]): a randomised controlled trial. Lancet Oncol 2006;7:719-727.

10 Pignon JP, Tribodet H, Scagliotti GV, et al.: Lung adjuvant cisplatin evaluation: a pooled analysis by the LACE Collaborative Group. J Clin Oncol 2008; 26:3552-3559.

11 NSCLC Meta-Analyses Collaborative Group, Arriagada $\mathrm{R}$, Auperin A, et al.: Adjuvant chemotherapy, with or without postoperative radiotherapy, in operable non-small-cell lung cancer: two metaanalyses of individual patient data. Lancet 2010; 375:1267-1277.

12 Pisters KM, Vallières E, Crowley JJ, et al.: Surgery with or without preoperative paclitaxel and carboplatin in early-stage non-small-cell lung cancer: Southwest Oncology Group Trial S9900, an intergroup, randomized, phase III trial. J Clin Oncol 2010;28:1843-1849.

13 Scagliotti GV, Pastorino U, Vansteenkiste JF, et al.: Randomized phase III study of surgery alone or surgery plus preoperative cisplatin and gemcitabine in stages IB to IIIA non-small-cell lung cancer. J Clin Oncol 2012;30:172-178.
14 Felip E, Rosell R, Maestre JA, et al.: Preoperative chemotherapy plus surgery versus surgery plus adjuvant chemotherapy versus surgery alone in earlystage non-small-cell lung cancer. J Clin Oncol 2010; 28:3138-3145.

15 Olaussen KA, Dunant A, Fouret P, et al.: DNA repair by ERCC1 in non-small-cell lung cancer and cisplatin-based adjuvant chemotherapy. N Engl J Med 2006;355:983-991.

16 Filipits M, Pirker R, Dunant A, et al.: Cell cycle regulators and outcome of adjuvant cisplatin-based chemotherapy in completely resected non-smallcell lung cancer: the International Adjuvant Lung Cancer Trial Biologic Program. J Clin Oncol 2007; 25:2735-2740.

17 Pirker R, Filipits M, Dunant A, et al.: IALT-Bio: a challenging research to improve adjuvant chemotherapy of completely resected NSCLC. J Thorac Oncol 2007;2:S397-S398.

18 Pirker R, Rousseau V, Paris E, et al.: LACE-Bio: Cross-validation and pooled analyses of the putative prognostic/predictive biomarkers p27, p16 and cyclin E in IALT, ANITA, JBR10 and CALGB 9633. J Thorac Oncol 2010;5:S503.

19 Zhu CQ, Ding K, Strumpf D, et al.: Prognostic and predictive gene signature for adjuvant chemotherapy in resected non-small-cell lung cancer. J Clin Oncol 2010;28:4417-4424.

20 http://clinicaltrials.gov/ct2/show/NCT00478699? term $=S C A T+$ adjuvant\&rank $=1$.

21 http://clinicaltrials.gov/ct2/show/NCT00775385? term $=$ TASTE+adjuvant \& rank $=1$ 